\title{
Saccharin aversion acquired during unilateral spreading depression
}

\author{
JOEL L. DAVIS \\ Northern Illinois University, DeKalb, Ilinois 60115
}

\begin{abstract}
During unilateral cortical spreading depression (CSD), rats were given the chance to associate the flavor of saccharin $(5 \%)$ with the sickness produced by intraperitoneal injection of $\mathrm{LiCl}(100 \mathrm{mg} / \mathrm{kg})$. When offered a choice between saccharin and water on the next day, the animals preferred water when tested with the trained $(67 \%)$ or untrained $(73 \%)$ hemisphere. Controls receiving no LiCl displayed saccharin preference $(80 \%)$. Like the pain motivated passive avoidance, saccharin aversion cannot be lateralized by the functional split-brain technique.
\end{abstract}

Learning under unilateral cortical spreading depression (CSD) leads to formation of memory traces which are either confined to the intact hemisphere (instrumental somatic relations) or are bilaterally stored at some subcortical level (classically conditioned visceral reactions). One possibility for predicting a unilateral or bilateral storage mode might be found in different learning tasks. For example, Megirian and Bures (1970) have suggested that the effector (somatic vs visceral) may be important, whereas Ross and Russell (1967) stressed the significance of the learning paradigm. The nature of the CS and UCS is another variable which should be investigated in more detail.

Classical visceral conditioning (Garcia \& Ervin, 1968; Nachman, 1970) leads to strong avoidance of the flavor of food or liquids ingested during the several hours before lithium chloride administration. Although the ultimate result is passive avoidance, it is based on a UCS and a CS that is somewhat unique in traditional passive avoidance paradigms. Davis and Bures (1972) found that saccharin aversion develops when bilateral CSD is elicited after saccharin drinking but shortly before (15 min) injection of $\mathrm{LiCl}$, which would suggest that the memory trace is not stored at the cortical level. Additionally, pain motivated avoidance learning acquired during unilateral CSD is not lateralized (Bures, Buresova, and Fifkova, 1964), and is probably stored subcortically. This study was designed to determine whether, as in the pain motivated passive avoidance, saccharin aversion would not be lateralized by a functional split-brain technique.

\section{METHOD}

The subjects were 36 adult male hooded rats (weight $225 \mathrm{~g}$ ) of the Long-Evans strain. They were housed in individual cages and had free access to food throughout the experiment. The testing apparatus consisted of a rectangular chamber $(50 \times 25 \mathrm{~cm})$ into which projected two glass drinking tubes $2.5 \mathrm{~cm}$ apart. Each tube was connected to a graduated $75 \mathrm{ml}$ pipette containing either $0.5 \mathrm{~g} / 1$ sodium saccharin or water.

During the four experimental days the rats received only those liquids drunk in the testing chamber. All rats were deprived of water $24 \mathrm{~h}$ before being placed in the test box for $15 \mathrm{~min}$ on day one. Water was in both tubes at this time. The 15-min water access period was repeated on day two. Four hours after the second session the animals were anesthetized with ether and trephine holes ( $4 \mathrm{~mm}$ in diameter) were made over both parietal areas. A plastic ring (10 $\mathrm{mm}$ high, $12 \mathrm{~mm}$ in ternal diameter) was sutured to the skull about the holes, filled with saline soaked cotton and closed with a plastic screw cap. Unilateral CSD was elicited five minutes before the third and fourth sessions. The cap and cotton were removed and a piece of filter paper $(3 \times 3 \mathrm{~mm})$ soaked with $25 \% \mathrm{KCl}$ was applied to the exposed dura of one hemisphere. The presence of CSD was confirmed by the loss of placing reactions in the limbs contralateral to the depressed hemicortex.

All subjects with CSD in the right hemisphere were given 15 -min access to saccharin $(0.6 \mathrm{~g} / 1)$ in both tubes on day 3 . Groups $B$ and $C$ were injected 30 min later with lithium chloride $(100 \mathrm{mg} / \mathrm{kg}$ i.p.). Two hours later the filter paper was removed, the cortical surface was washed with saline, and the cotton pledget and cap were replaced. On day four CSD was elicited in the left hemisphere in groups $A$ and $B$ and in the right hemisphere in group C. The subjects were allowed to choose between saccharin in one and water in the other drinking tube. The amount of the fluids was measured with $0.1 \mathrm{ml}$ accuracy. The results were evaluated using conventional statistical methods.

The animals drank equally from both tubes on days one and two. The average water consumption over the 15-min water access period was $8.2 \mathrm{ml}$. The rats were then assigned to groups $\mathrm{A}, \mathrm{B}$, and $\mathrm{C}$, which were matched for water intake on days one and two. Saccharin consumption on day three dropped to $7.6 \mathrm{ml}$, probably due to slight adipsia caused by unilateral CSD.

\section{RESULTS}

The overall fluid intake on day 4 was slightly lower in groups $\mathrm{A}$ and $\mathrm{B}(4.7 \pm 0.5 \mathrm{ml})$ than group $\mathrm{C}$ $(6.1 \pm 0.3 \mathrm{ml})$. The results of the saccharin-water choice are illustrated in Figure 1. As expected, the control group $\mathbf{A}$ which had received no $\mathrm{LiCl}$ on day three displayed marked preference for saccharin. Group $C$ in which left CSD was evoked both on days three and four showed the expected preference for water. The same preference was observed in group B, tested with the naive left hemisphere which did not participate in the saccharin-lithium pairing on the previous day. The difference between group $\mathrm{C}$ and groups $\mathrm{A}$ and $\mathrm{B}$ were significant for $\mathrm{p}<.001$. Animals injected with $\mathrm{LiCl}$ 


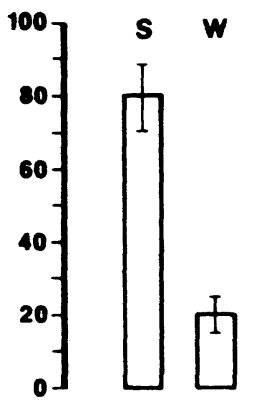

A

B
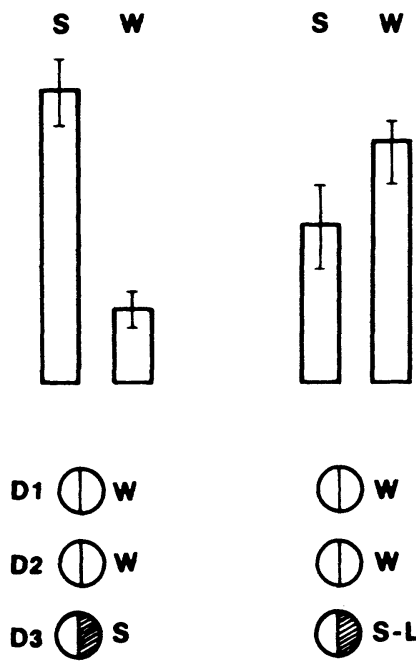

D4 Sw
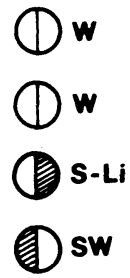

C
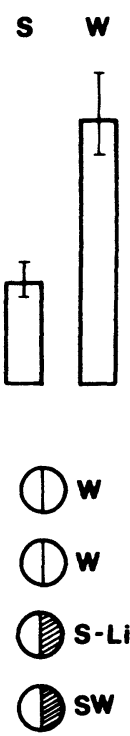

Figure 1. Percentage of saccharin (S) or water $(W)$ consumed on day four (above). SEM indicated by vertical bars. Below: experimental paradigm. $D_{1}-D_{4}$ : days one to four. LI: lithium injection. $S$ and $W$ indicate the solutions available in the drinking tubes. CSD denoted by shading.

displayed characteristic symptoms of gastrointestinal distress.

\section{DISCUSSION}

Results of the present experiments indicate that the saccharin aversion acquired during unilateral CSD cannot be lateralized. Although the learning procedure yielded a satisfactory memory trace in the left hemisphere (group C), the naive right hemisphere's preference for saccharin on day four was quite dependent on whether the left hemisphere was (group B) or was not (group A) exposed to the saccharin-lithium association on day three. The neural mechanisms of saccharin aversion are thus not clearly different from those of the pain motivated passive avoidance which can be well retrieved with the untrained hemisphere. This similarity can hardly be accounted for by the properties of the unconditioned stimuli. The visceral pain and nausea caused by $\mathrm{LiCl}$ are represented in the brain perhaps more diffusely that the somatic pain.

The inability to lateralize the saccharin aversion indicates that flavor is not a unique type of CS, at least for passive avoidance tasks. Whereas in most passive avoidance tasks the CS is the entire situation, consisting of complex visual, somatosensory, auditory, or olfactory stimuli, the only relevant cue in the saccharin aversion experiments is the taste of the ingested solution. Since taste thresholds increase about 10 times after surgical (Benjamin \& Pfaffman, 1955) or functional (Janebova, 1967) ablation of the gustatory cortex in rats, this brain region should be necessary for detection of low concentrations of a stimulus substance in solution. Nonetheless, it would seem unlikely from this study and others (Davis and Bures, 1972) that the memory trace is stored at the cortical level. A subcortical trace can mediate learning in functionally decorticated rats provided that saccharin was administered to intact animals. The fact that lateralization of saccharin aversion does not occur may indicate that the engram must exist outside the intact hemicortex and most probably as some subcortical memory trace. In addition, the nonlateralization of saccharin aversion is not unexpected if one assumes the paradigm to be similar to other aversive reactions of the passive inhibitory type which are also nonlateralized.

\section{REFERENCES}

Benjamin, R. M., \& Pfaffman, C. Cortical localization of taste in albino rat. Journal of Neurophysiology, 1955, 56-64.

Bures, J., Buresova, O., \& Fifkova, E. Interhemispheric transfer of a passive avoidance reaction. Journal of Comparative and Phy siological Psy chology, 1964, 57, 326-330.

Buresova, O., Rudiger, W., Bures, J., \& Fifkova, E. The role of the hypothalamic drinking center in unconditioned and conditioned control of water intake. Physiologia Bohemoslovaca, 1962, 11, 492-496.

Davis, J. L., \& Bures, J. Disruption of saccharin aversion learning by cortical spreading depression in the CS-UCS interval. Journal of Comparative and Phy siological Psychology, 1972, 80, 398-402.

Garcia, J., \& Ervin, F. R. Gustatory-visceral and telereceptor-cutaneous conditioning: adaptation in internal and external milieus. Communications in Behavioral Biology, 1968, Part A, 1, 389-515.

Janebova, M. Cortical spreading depression as a means of analyzing the role of the thalamic gustatory area in taste discrimination in rats. Phy siologia Bohemoslovaca, 1967, 16, 18-22.

Megirian, D., \& Bures, J. Unilateral cortical spreading depression and conditioned eyeblink responses in the rabbit. Experimental Neurology, 1970, 73, 31-37.

Nachman, M. Limited effects of electroconvulsive shock on memory of taste stimulation. Joumal of Comparative and Phy siological Psychology, 1970, 73, 22-30.

Ross, R. B., \& Russell, J. S. Subcortical storage of classical conditioning. Nature, 1967, 214, 210-211.

(Received February 6, 1975.) 